\title{
Swiss Cheese Pattern a Harbinger of Dementia or an Incidental Finding in an Unusual Case?
}

\author{
Faheem Arshad* \\ Department of Neurology, National Institute of Mental Health and Neurosciences (NIMHANS), India \\ *Corresponding author: Faheem Arshad, Department of Neurology, National Institute of Mental Health and Neurosciences \\ (NIMHANS), Bengaluru, India
}

\begin{abstract}
Dilated Virchow Robin (VR) spaces are pial- line fluid filled structures which surround the walls of small penetrating vessels. In a severe form they develop a swiss cheese pattern or a cribriform pattern in straitum which may predispose to cognitive impairment. We report a patient with change in personality associated with diffuse atrophy, hypometabolism, microbleeds and swiss cheese striatum which is rare.
\end{abstract}

Keywords: Swiss cheese pattern; Dementia; Virchow robin space

Abbreviations: VR: Virchow Robin; MRI: Magnetic Resonance Imaging; FLAIR: Fluid Attenuated Inversion Recovery; CSF: Cerebrospinal Fluid

\section{Introduction}

Virchow Robin (VR) spaces are pial-lined, fluid filled, structures which surround the walls of small penetrating arterioles and venules as they course from subarachnoid space to brain parenchyma. These often appear in basal ganglia and centrum semi vale and are reflected in Magnetic Resonance Imaging(MRI) brain as hypointense in T1-weighted images, hyperintense on T2-weighted and hypointense on Fluid Attenuated Inversion Recovery(FLAIR) images, thus distinguished from pathological white matter lesions by persistent is intensity to Cerebrospinal Fluid(CSF) on all sequences, lack of enhancement and sharply defined margins. Dilated VR spaces can appear on neuroimaging as single enlarged cavity(up to $2 \mathrm{~cm}$ in diameter) or may appear as hundreds of bilateral 1-2 mm foci in the basal ganglia, subcortical white matter and sub insular area lateral to lentiform nucleus, a pattern sometimes referred to as etat crible or cribriform or Swiss cheese striatum.

Though a common finding in elderly population, studies have shown that enlarged VR spaces are a marker of small vessel disease and associated with incident dementia and depression. Multiple mechanisms for giant VR spaces have been described which include mechanical trauma due to CSF pulsation, fluid exudation due to abnormalities of the vessel wall abnormality and ischemic injury to perivascular tissue causing ex vacuo effect. The precise function of VR spaces is not completely understood. They are believed to serve as a lymphatic of brain also known as the glymphatic system whereby CSF exchanges with the interstitial fluid within the brain parenchyma, including clearing the interstitial solutes such as betaamyloid.

\section{Epidemiology}

Dilated Virchow robin spaces were described Durant-Fardel in 1843. In a study of healthy participants using high resolution images prevalence was $1.6 \%$. In radiological studies involving patients VR spaces were found in 3\% patients under 20 years of age. In addition, studies have shown higher rate of cognitive decline with dilated VR spaces, which is intriguing, and its elucidation may improve the complex understanding of role of vascular alterations and higher risk of cognitive decline [1]. Furthermore, dilated VR spaces have different topographically patterns in microangiopathies like cerebral amyloid angiopathy where they are mostly seen in centrum semiovale and hypertensive angiopathy where they are predominantly seen in basal ganglia [2]. To date, longitudinal data with regard to significance of dilated VR spaces in healthy older adults is scarce. However small number of studies have shown association with development of new onset dementia. 
Prevalence is more in vascular dementia compared to Alzheimer's dementia and healthy controls.

\section{Clinical presentation}

Increased basal ganglia or centrum semiovale perivascular spaces have been associated with worse nonverbal reasoning and visuospatial cognitive abilities. Various clinical presentations have been reported which include parkinsonism, hemisensory symptoms and in addition depending on the size of the VR space causing mass effect. Dilated VR spaces have been associated with vascular dementia and also have been correlated with reduced cognitive function [3]. Thus for a clinician differential diagnosis of dilated spaces should always be considered in view of multiple mimics like multiple lacunar infarctions, cryptococcosis, multiple sclerosis, mucopolysaccharidosis, cystic neoplasms and arachnoid cysts. Knowledge of their signal changes in neuroimaging may help in differentiating these lesions from dilated VR spaces.

\section{Clinical case}

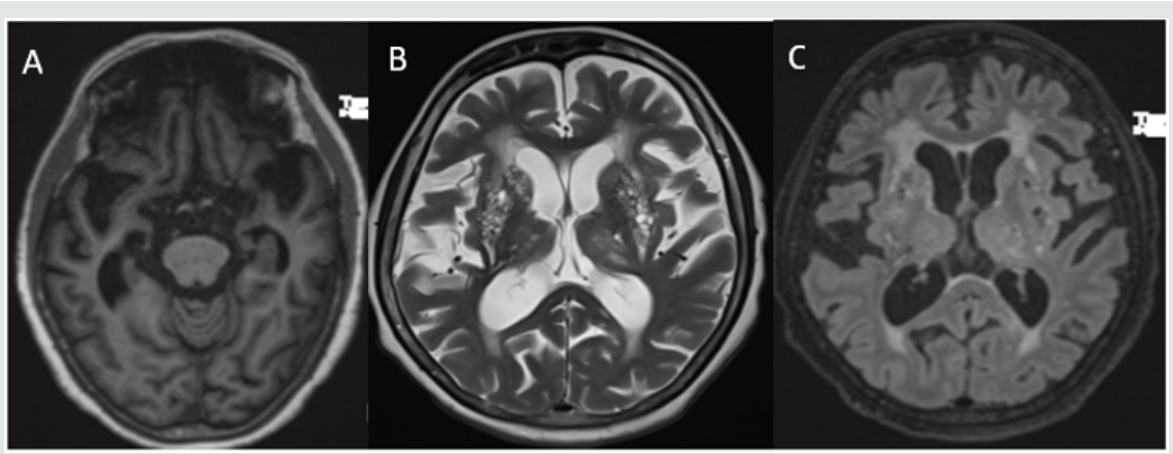

Figure 1: (A) T1-weighted MRI showed bilateral frontal and anterior temporal lobe atrophy; (B, C) T2/FLAIR hyperintensities showing confluent foci in the bilateral deep and periventricular white matter, and prominent perivascular spaces in bilateral basal ganglia suggestive of a swiss cheese pattern.
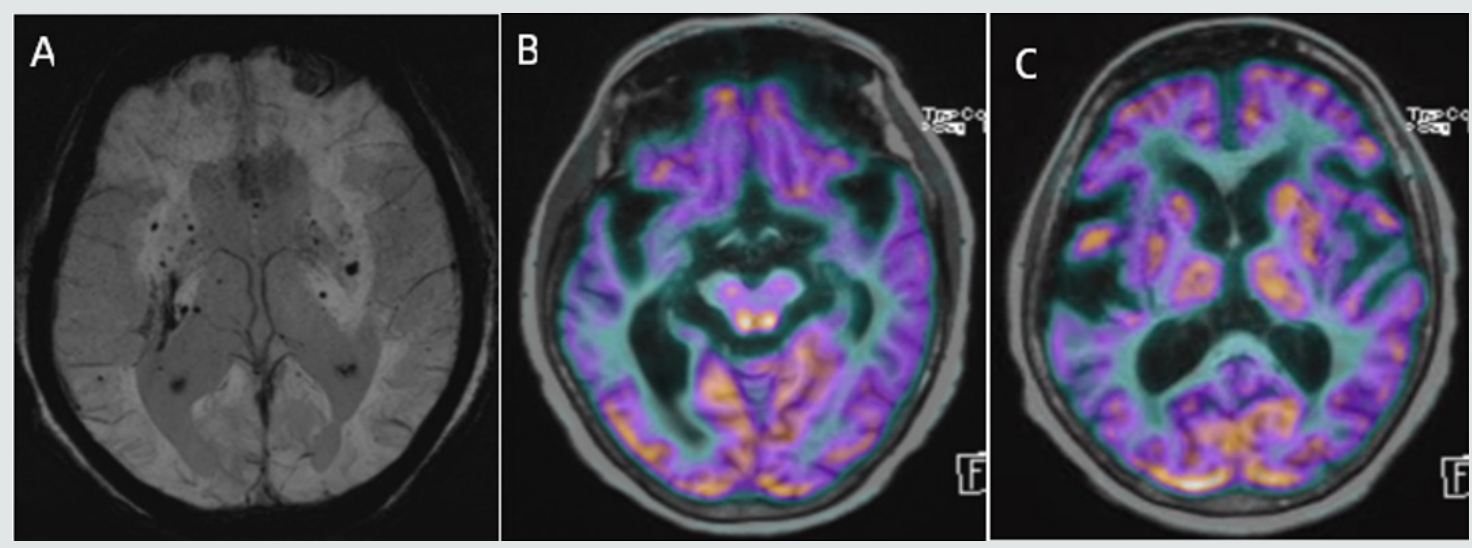

Figure 2: (A) SWI sequence showing multiple foci of blooming in bilateral basal ganglia, thalami, cerebral hemispheres, brainstem and left cerebellar hemisphere. (B) Positron emission tomography (PET) MRI brain showing hypometabolism in bilateral parietal lobes, medial and anterior temporal lobes and orbitofrontal cortex. (C) PET MRI brain showing hypometabolism in dilated VR spaces in the striatum.

60 year old female, presented with the 5 years history of change in personality associated with behavioural disturbances in the form of getting angry for trivial issues and screaming at family members, trying to pick things in front of her, telling that insects are crawling on her clothes. Since last 2 years caregivers report history of fluctuating restlessness, wandering aimlessly, muttering to self, disinhibited behaviour and apathy. Since last 6 months she had lost concern for family members, started becoming slow in her activities especially walking and became completely dependent for her daily activities of living. There was also history of incontinence without any concern. There was no history of food faddism, utilization behaviour, myoclonic jerks, seizure, recurrent falls, bulbar symptoms, weight loss, change in bowel habits. Patient was diagnosed case of hypertension since 5 years and was on medications. She was not cooperative for mental status examination. Her physical examination revealed mild bradykinesia, brisk reflexes, mildly wide based gait with reduced clearance and primitive reflexes were present. Blood investigation were all normal. MRI brain T1-weighted image (Figure 1A) showed bilateral frontal and anterior temporal lobe atrophy, confluent foci of T2/FLAIR hyperintensities (Figure1B and C) in the bilateral deep and periventricular white matter and T2 weighted image showed prominent perivascular spaces in bilateral basal ganglia suggestive of a swiss cheese pattern. In addition, there were multiple foci of blooming on Susceptible Weighted Images (SWI) (Figure 2A) are seen in the bilateral basal ganglia, thalami, 
cerebral hemispheres, brainstem and left cerebellar hemisphere. Positron Emission Tomography (PET) MRI brain (Figure 2B) showed hypometabolism in bilateral parietal lobes, medial and anterior temporal lobes and orbitofrontal cortex. PET MRI brain (Figure 2C) showed hypometabolism in dilated VR spaces in the striatum. Final diagnosis of mixed dementia associated with swiss cheese brain syndrome was considered and patient was managed as per standard guidelines for dementia. In addition to Vascular dementia, more commonly mixed dementia includes Alzheimer's dementia, however patient had predominant features suggestive of Frontotemporal dementia which less commonly associated with microbleeds and dilated VR spaces as seen in this patient. Whether it is a combination of multiple dementias contributed by enlarged VR spaces will remain unanswered.

\section{Conclusion}

Dilated VR spaces may be an either an incidental finding on MRI without any pertinent manifestations. However, evidence suggests that these may be predisposing risk factor for cognitive impairment. Whether estimating the burden of perivascular spaces may help in predicting development of cognitive impairment and type of dementia, remains undetermined and may require further research.

\section{References}

1. Zhu YC, Dufouil C, Soumare A, Mazoyer B, Chabriat H, et al. (2010) High degree of dilated virchow-robin spaces on mri is associated with increased risk of dementia. J Alzheimers Dis 22(2): 663-672.

2. Charidimou A, Boulouis G, Pasi M, Auriel E, van Etten ES, et al. (2017) MRI-visible perivascular spaces in cerebral amyloid angiopathy and hypertensive arteriopathy. Neurology 88(12):1157-1164.

3. Burnett MS, Witte RJ, Ahlskog JE (2014) Swiss cheese striatum: Clinical implications. JAMA neurology 71(6): 735-741.
(C) (P) This work is licensed under Creative

To Submit Your Article Click Here: Submit Article

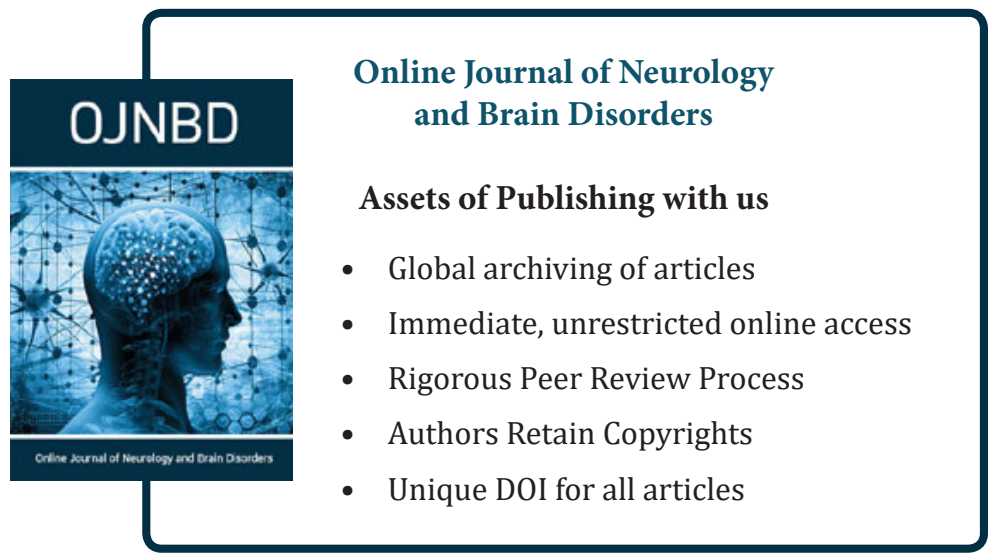

